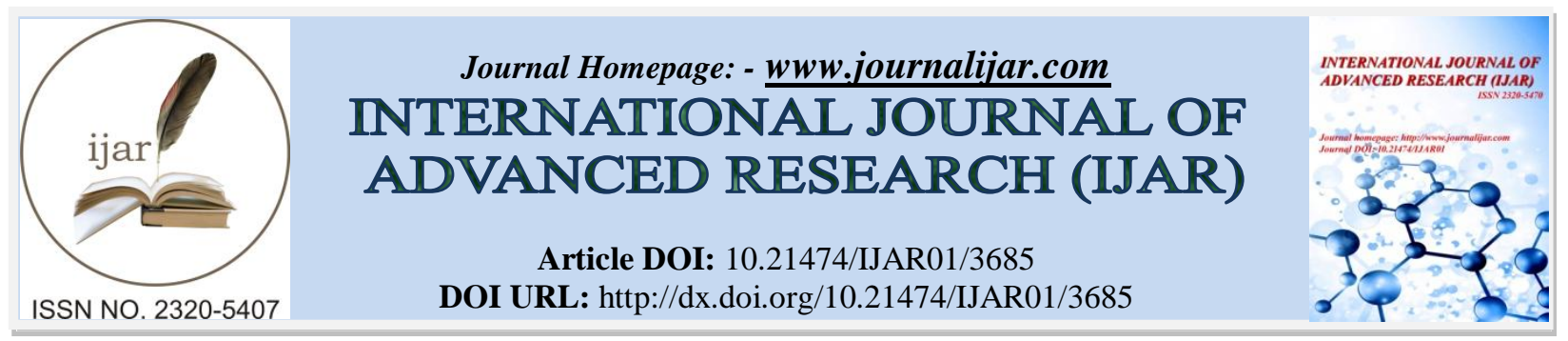

RESEARCH ARTICLE

\title{
DETERMINATION OF RESIDUAL SOLVENTS IN NEOSTIGMINE METHYLSULFATE BY HEADSPACE GAS CHROMATOGRAPHY.
}

\author{
Dr. Sayyed Hussain ${ }^{1}$, Dr. Amit Gosar ${ }^{2}$ and Tabrez Shaikh ${ }^{1,2^{*}}$.
}

1. Sir Sayyed College of Arts, Commerce and Science, P.G. Department of Chemistry, Roshangate, Aurangabad, 431001 Maharashtra, India.

2. Indoco Remedies Limited, Analytical research \& development department, Navimumbai, 400701, Maharashtra, India.

\section{Manuscript Info}

(.........................

Manuscript History

Received: 13 January 2017

Final Accepted: 01 February 2017

Published: March 2017

Key words:-

Headspace gas chromatography,

Neostigmine Methylsulfate, residual

solvent, validation

\section{Abstract}

A static headspace gas chromatographic (GC-HS) method is developed for quantitative determination of residual organic solvents such as acetone, ethyl acetate and acetonitrile used in the synthesis of Neostigmine Methylsulfate. Separation was achieved on capillary column DB-624 of length $30 \mathrm{~m}$ having internal diameter $0.32 \mathrm{~mm}$ and $1.8 \mu \mathrm{m}$ film thickness using a Flame ionization detector with gradient column oven temperature program. The headspace condition was optimized with the vial equilibration temperature of $100^{\circ} \mathrm{C}$ and time at $30 \mathrm{~min}$. The injection was carried out in split mode, with a split ratio of 10:1. N-methyl-2-pyrrolidone is selected as a diluent to obtain good sensitivity along with the recovery. Analytical method validation was performed within the requirements of ICH validation guidelines Q2 (R1). System suitability parameter set with tailing factor, theoretical plate and relative standard deviation as required with various pharmacopoeias are checked. Limits of detection and quantitation, precision, linearity and accuracy along with robustness are performed, and acceptable results are obtained. The proposed developed method is demonstrated to be simple, sensitive, linear, accurate and robust, hence can be used to determine the residual organic solvents in Neostigmine methylsulfate drug substance.

Copy Right, IJAR, 2017,. All rights reserved.

\section{Introduction:-}

Neostigmine methylsulfate is a acetylcholinesterase inhibitor used in the treatment of myasthenia gravis and to reverse the effects of muscle relaxants such as gallamine and tubocurarine and is currently recommended by the WHO for the treatment of neurotoxic snakebite [1-4].

In the synthesis of drug substances various organic solvents are used at various stages. These solvent may be used as a medium for the reaction or purification of intermediates or drug substances. Intermediates and drug substances are dried at a specific temperature to remove the used solvents, but traces of them may carry forward to drug substance as impurities which are called as residual solvents.

Corresponding Author:- Tabrez Shaikh.

Address:- Sir Sayyed College of Arts, Commerce and Science, P.G. Department of Chemistry,

Roshangate, Aurangabad, 431001 Maharashtra, India. 
Residual solvents are unwanted chemical which does not have any no therapeutic benefit and in some cases they are toxic. Based on their toxicity, they are classified into three categories Class 1 solvents are known or suspected to be carcinogens to human and environmental hazards, hence these should be avoided in the production of drug substances, unless their use is strongly justified in a risk-benefit assessment. Class 2 solvents are non-mutagenic and known or suspected to be carcinogens in animals, therefore these solvents should be limited in order to ensure patient safety. Class 3 solvents with low toxic potential to humans; no health based exposure limit is needed. Residual solvents are generally determined using Headspace gas chromatography (GC-HS) techniques. Analytical methods used for determination of residual solvent should be validated as per ICH guidelines. The aim of this study is to develop and validate the GC-HS method of analysis for residual solvents namely acetone, ethyl acetate and acetonitrile in Neostigmine methylsulfate. Some of the methods for identification of residual solvent in drugs are also discussed [5-9].<smiles>COS(=O)(=O)[O-]</smiles>

\section{Neostigmine Methylsulfate}

Fig. 1:- Structure of Neostigmine methylsulfate

Table 1:- Residual solvent with there class and limits.

\begin{tabular}{|c|c|c|}
\hline Solvent & Class & Limit (ppm) \\
\hline Acetone & 3 & 5000 \\
\hline Ethyl acetate & 3 & 5000 \\
\hline Acetonitrile & 2 & 410 \\
\hline
\end{tabular}

\section{Materials:-}

\section{Reagent and Chemicals:-}

Neostigmine Methylsulfate sample was received from Analytical research and development department of Indoco research centre (Navi Mumbai). GC grade Acetone, Ethyl acetate and Acetonitrile were purchased from Merck (India). N-methyl-2-pyrrolidone used as diluent for preparation of sample and standard was purchased from Spectrochem.

\section{Instrumentation:-}

Perkin Elmer, Clarus 500 gas chromatographic system with a Turbo matrix headspace gas sampler and Flame ionization detector (FID) was used for separation and detection of solvents. Sartorius (Germany) analytical balance was used for weighing the materials.

\section{Methodology:-}

\section{Method optimization:-}

Various GC columns such as DB-Wax, DB-1 and DB-5 were used of various dimensions, but the best separation was achieved on DB-625 (Make-Agilent) with dimension $30 \mathrm{~m}$ length and $0.32 \mathrm{~mm}$ of internal diameter and coated with stationary phase of $1.8 \mu \mathrm{m}$ film thickness. Details of other optimized gas chromatographic and headspace parameters are given in Table 2 and Table 3 respectively. For suitability of a system, tailing factor kept was not more than 2.0, theoretical plate not less than 5000 and \% RSD not more than 5.0\% was kept for peak area of each solvent for six standard solution replicate injections. 
Table 2:- Optimized gas chromatographic conditions.

\begin{tabular}{|l|l|}
\hline Detector & Flame Ionization Detector \\
\hline Column oven temperature program & Initial $40^{\circ} \mathrm{C}$, hold for 8.0 minutes \\
\cline { 2 - 2 } & Increase $@ 20^{\circ} \mathrm{C}$ per minute to $200^{\circ} \mathrm{C}$ \\
\cline { 2 - 2 } & Hold at $200^{\circ} \mathrm{C}$ for 4.0 minutes \\
\hline Detector Temperature & $220^{\circ} \mathrm{C}$ \\
\hline Injector Temperature & $200^{\circ} \mathrm{C}$ \\
\hline Attenuation & -6 \\
\hline Split Ratio & $10: 01$ \\
\hline Carrier Gas & Nitrogen \\
\hline Carrier Gas Flow & $1.50 \mathrm{~mL} / \mathrm{min}$ \\
\hline Run time & $20 \mathrm{~min}$ \\
\hline Range & 01 \\
\hline
\end{tabular}

Table 3:- Optimized headspace conditions.

\begin{tabular}{|c|c|}
\hline Oven equilibration temperature & $100^{\circ} \mathrm{C}$ \\
\hline Needle temperature & $110^{\circ} \mathrm{C}$ \\
\hline Transfer line temperature & $120^{\circ} \mathrm{C}$ \\
\hline Thermostat time/Vial incubation time & 30.0 minutes \\
\hline Pressurization time & 3.0 minutes \\
\hline Injection time & 0.05 minutes \\
\hline Withdrawal time & 0.5 minutes \\
\hline GC cycle time & 34.0 minutes \\
\hline
\end{tabular}

\section{Preparation of solutions:-}

\section{Blank solution:-}

Transferred $1.0 \mathrm{~mL}$ of diluent into headspace vials and sealed the vials immediately using PTFE septa with aluminium crimp cap.

\section{Standard stock solution:-}

Transferred $5.0 \mathrm{~g}$ of Acetone, $0.410 \mathrm{~g}$ of Acetonitrile and $5.0 \mathrm{~g}$ of Ethyl acetate into a $100 \mathrm{~mL}$ volumetric flask containing about $20 \mathrm{~mL}$ diluent and made up to mark with diluent.

\section{Standard solution:-}

Transferred $1.0 \mathrm{~mL}$ standard stock solution into a $100 \mathrm{~mL}$ volumetric flask and made upto mark with diluent. Transferred $1.0 \mathrm{~mL}$ of diluted solution into headspace vials and sealed the vial immediately using PTFE septa with aluminium crimp cap.

Test solution:-

Transferred $0.10 \mathrm{~g}$ sample into a headspace vial, added $1.0 \mathrm{~mL}$ of diluent and sealed the vial immediately using PTFE septa with aluminium crimp cap.

\section{Procedure:-}

Conditioned the column at $200^{\circ} \mathrm{C}$ and then equilibrated at $40^{\circ} \mathrm{C}$. Injected blank solutions, six standard solution and test solution. Recorded the peak area of each solvent and calculated solvent content in Neostigmine Methylsulfate by formula below

Ethyl acetate/Acetone/Acetonitrile $(\mathrm{ppm})=(\mathrm{AT} / \mathrm{AS}) \times(\mathrm{WS} / \mathrm{WT}) \times 100$

Where, AT is area of solvent in the test sample, AS is average area of corresponding solvent in the standard solution, WS is weight of corresponding solvent taken for standard solution preparation $(\mathrm{g})$ and WT is weight of Neostigmine Methylsulfate test sample (g). 


\section{Analytical Method Validation:-}

The analytical method validation work was conducted according to the ICH (International Conference on Harmonization) guidelines and with reference to other literature [10-16]. The parameter with which analytical method is validated is Specificity, Limit of detection, Limit of quantitation, Linearity, Accuracy, Precision and Robustness.

\section{Specificity:-}

Capability of the method to measure the analyte peak (solvent) response in the presence of other components is termed as specificity. For this, blank, standard, test sample and spiked test sample solutions were injected and observed the chromatogram for any interference from blank and test sample peaks at a retention time of solvent peaks. It was observed that there was not interference at a retention time of solvent peaks (Fig. 3). Also the retention time of the solvent peaks for standard and the spiked test sample were matching (Table 4).

Table 4:- Retention time of solvent peaks

\begin{tabular}{|c|c|c|}
\hline \multirow{2}{*}{ Solvent } & \multicolumn{2}{|c|}{ Retention time (min) } \\
\cline { 2 - 3 } & Standard solution & Spiked test sample \\
\hline Acetone & 4.280 & 4.276 \\
\hline Acetonitrile & 4.804 & 4.801 \\
\hline Ethyl acetate & 8.592 & 8.592 \\
\hline
\end{tabular}

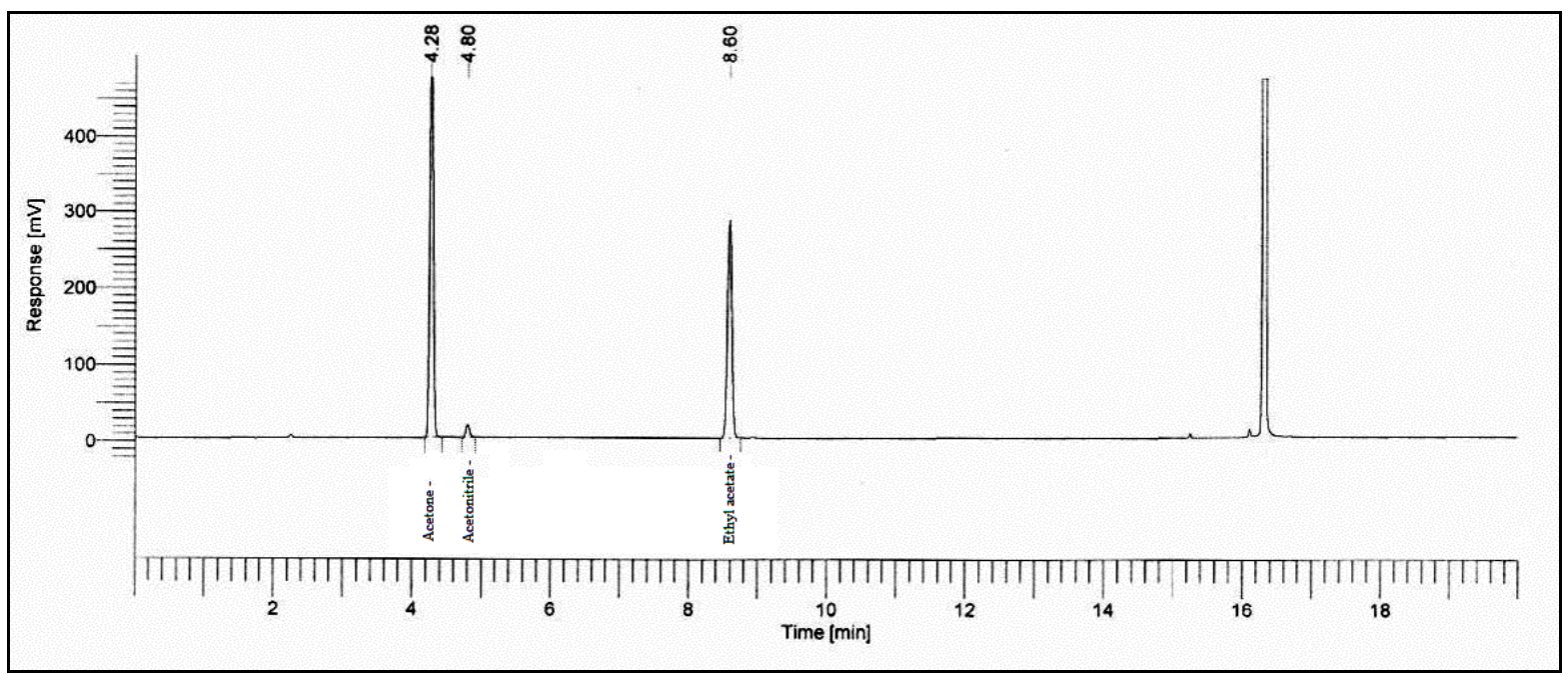

Fig 3:- Neostigmine Methylsulfate sample spiked with solvents.

\section{Limit of detection (LOD) and Limit of quantitation (LOQ):-}

For the establishment of LOD and LOQ, six standard solutions of known concentration were injected and average signal-to-noise ratio for solvent peak was determined. Concentration for each solvent was calculated for which signal to noise is 3 and 10 and reported as the limit of detection and limit of quantitation respectively (Table 5).

Limit of detection $(\mathrm{ppm})=3 \times \mathrm{C} / \mathrm{S}$

Limit of quantitation $(\mathrm{ppm})=10 \times \mathrm{C} / \mathrm{S}$

Where $\mathrm{C}$ is the concentration of solvent in standard solution ( $\mathrm{ppm}$ ) and $\mathrm{S}$ is the signal to noise ratio of that solvent peak.

Table 5:- Detection and quantitation limits.

\begin{tabular}{|c|c|c|}
\hline Solvent & Limit of detection $(\mathrm{ppm})$ & Limit of quantitation $(\mathrm{ppm})$ \\
\hline Acetone & 3.97 & 13.23 \\
\hline Acetonitrile & 9.49 & 31.65 \\
\hline Ethyl acetate & 7.87 & 26.23 \\
\hline
\end{tabular}




\section{Linearity:-}

Six linearity solutions were prepared ranging from LOQ to $150 \%$ of limit level concentration of each solvent. Injected each solution in triplicate and calculated average peak area for each solvent. Linearity curves were drawn by plotting the graph of the average peak area of solvent against its concentration in ppm in linearity solutions (Fig. 4-6). Reported slope, intercept, \% y intercept, regression coefficient $\left(\mathrm{r}^{2}\right)$ and range for which linearity is established (Table 6).

Table 6:- Linearity and range of solvents.

\begin{tabular}{|c|c|c|c|c|c|}
\hline Solvent & Slope & Intercept & \% y intercept & $\mathrm{r}^{2}$ & Range (ppm) \\
\hline Acetone & 367.78 & 2621.6 & 0.14 & 1.0000 & 13.23 to 7514.58 \\
\hline Acetonitrile & 147.41 & 458.64 & -0.76 & 0.9999 & 31.65 to 624.65 \\
\hline Ethyl acetate & 232.83 & 2226.1 & 0.19 & 1.0000 & 26.57 to 7547.85 \\
\hline
\end{tabular}

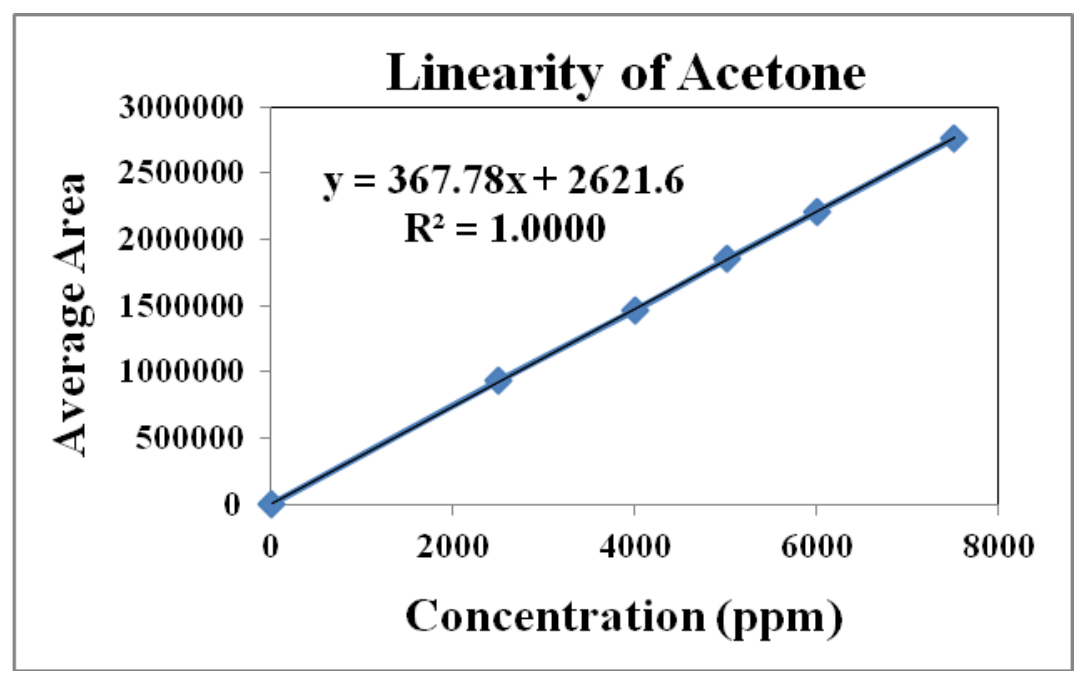

Fig. 4:- Linearity plot for Acetone.

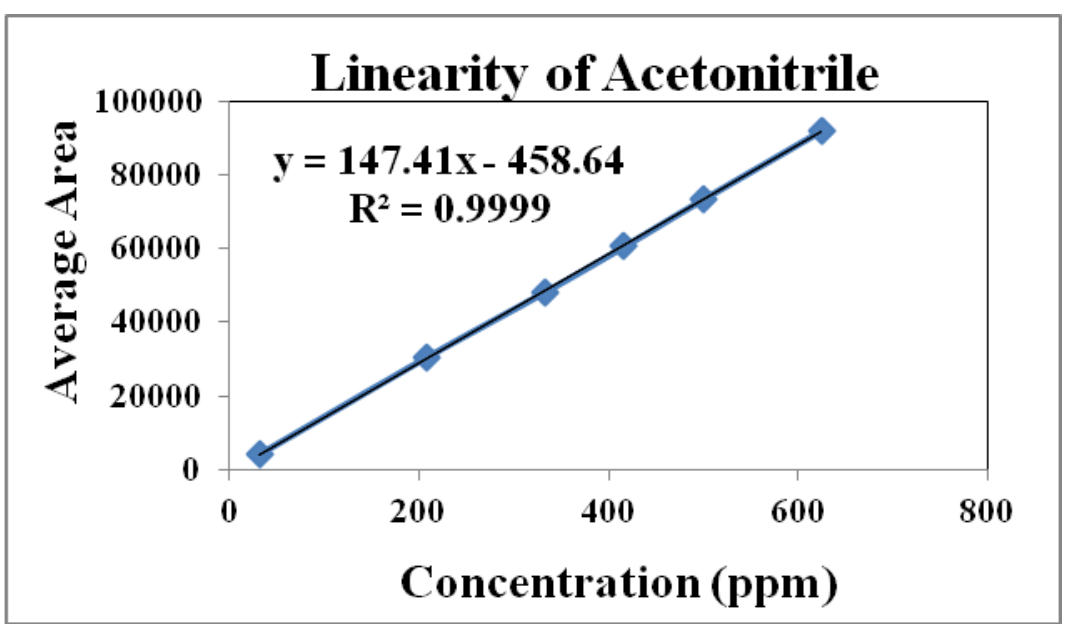

Fig. 5:- Linearity plot for Acetonitrile. 


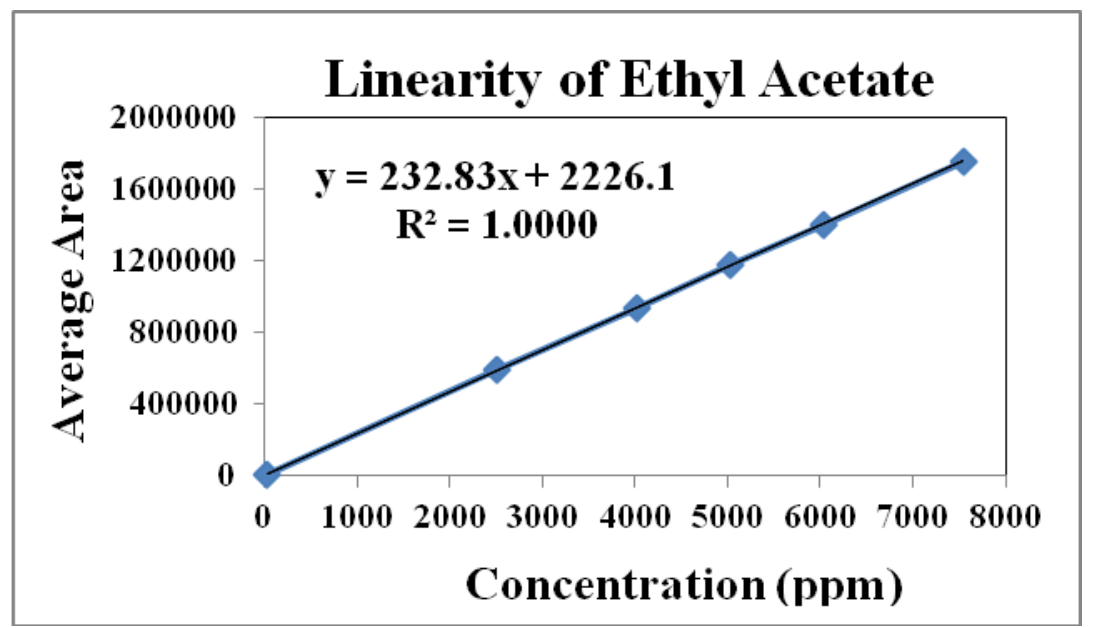

Fig. 6:- Linearity plot for Ethyl acetate

\section{Accuracy:-}

Accuracy was established by performing the recovery studies were each solvent was spiked in the test sample at four different concentration levels, i.e. LOQ, 80, 100 and $120 \%$ of target concentration. Each level was prepared in triplicate. The solvent content for each accuracy sample was calculated as per methodology and reported as observed concentration. To calculate the recovery for solvent, the ratio is taken of the observed solvent content and the theoretical solvent content spiked. Calculated percent accuracy for each sample and reported the average for each level. Recovery for each solvent was between $80 \%$ and $120 \%$ (Table7).

Table 7:- Recovery of Acetone, Acetonitrile and Ethyl acetate.

\begin{tabular}{|c|c|c|c|}
\hline \% Recovery & Acetone & Acetonitrile & Ethyl acetate \\
\hline LOQ & 94.79 & 109.51 & 103.68 \\
\hline $80 \%$ & 95.32 & 92.54 & 100.77 \\
\hline $100 \%$ & 98.14 & 93.19 & 104.03 \\
\hline $120 \%$ & 94.97 & 88.43 & 100.75 \\
\hline
\end{tabular}

\section{Precision:-}

System precision was carried out by analysing six standard solutions prepared as per methodology. \% RSD for the peak area for each solvent was found to be less than $5.0 \%$. Six samples were prepared by spiking each solvent with LOQ concentration and RSD for peak area was calculated which was less than $5.0 \%$. For repeatability (Inter-day) and intermediate precision (Intra-day and Inter-day), six test solutions were prepared by spiking the solvents at limit level concentration. RSD observed for each solvent content for repeatability and intermediate precision solution was less than $5.0 \%$ also cumulative RSD for repeatability and intermediate precision was less than $5.0 \%$.

Table 8:- Precision for acetone, acetonitrile and ethyl acetate.

\begin{tabular}{|c|c|c|c|}
\hline \multirow{2}{*}{ Parameter } & \multicolumn{3}{|c|}{ \% RSD for peak area } \\
\cline { 2 - 4 } & Acetone & Acetonitrile & Ethyl acetate \\
\hline System precision (Standard solution) & 1.56 & 1.53 & 1.55 \\
\hline Precision at LOQ & 1.30 & 1.56 & 1.36 \\
\hline Repeatability (Intra-day) & 0.64 & 0.96 & 0.58 \\
\hline Intermediate precision (Inter-day) & 0.61 & 1.01 & 0.68 \\
\hline Cumulative (Intra-day and Inter-day) & 0.64 & 1.54 & 0.67 \\
\hline
\end{tabular}

\section{Robustness:-}

For robustness, two deliberate changes were done with respect to carrier gas flow rate and vial incubation temperature. Each change consists of one upper set and one lower set (Table 9). For each set, three preparations were done by spiking the solvents in the test sample at limit level and analysed. \% RSD for each solvent content individually for three determinations and cumulative with six determinations of repeatability was observed for robustness solutions. RSD was found to be less than $5.0 \%$ (Table 10). 
Table 9:- Robustness parameter changes.

\begin{tabular}{|l|l|l|}
\hline Changes & Lower set & Upper set \\
\hline Change in carrier gas flow rate & $1.4 \mathrm{~mL} / \mathrm{min}$ & $1.6 \mathrm{~mL} / \mathrm{min}$ \\
\hline Change in vial incubation temperature & $95^{\circ} \mathrm{C}$ & $105^{\circ} \mathrm{C}$ \\
\hline
\end{tabular}

Table 10:- Robustness parameter changes.

\begin{tabular}{|l|l|l|l|l|l|l|}
\hline \multirow{2}{*}{ Sample ID } & \multicolumn{2}{l|}{ Individual \% RSD } & \multicolumn{2}{l|}{ Cumulative \% RSD } \\
\cline { 2 - 7 } & Acetone & Acetonitrile & Ethyl acetate & Acetone & Acetonitrile & Ethyl acetate \\
\hline Robustness-1 & 0.53 & 1.26 & 0.43 & 0.82 & 0.99 & 0.84 \\
\hline Robustness-2 & 0.59 & 1.13 & 0.44 & 0.61 & 1.19 & 0.54 \\
\hline Robustness-3 & 0.27 & 0.13 & 0.17 & 0.90 & 0.79 & 0.84 \\
\hline Robustness-4 & 1.28 & 0.48 & 1.26 & 0.90 & 0.96 & 1.13 \\
\hline
\end{tabular}

\section{Result and Discussion:-}

The retention time of the solvent peak of standard solution matches with that of the spiked test sample solution. No interference was observed at a retention time of the solvent peak from blank and test sample. The percentage recovery obtained for each solvent was in the range of $80 \%$ to $120 \%$, which is within ICH acceptance. Precision parameter shows the RSD was less than $5.0 \%$ for all the solvents in system precision, repeatability and intermediate precision at $100 \%$ concentration. Linearity was observed in the concentration range of LOQ to $150 \%$ with $\mathrm{r}^{2}$ values greater than 0.999 and y-intercept less than 5.0\% showing a good correlation between the response and solvent concentration. The calculated limit of detection and limit of quantitation for Acetone was $3.97 \mathrm{ppm}$ and $13.23 \mathrm{ppm}$, Acetonitrile was $9.49 \mathrm{ppm}$ and $31.65 \mathrm{ppm}$ and for Ethyl acetate $7.87 \mathrm{ppm}$ and $26.23 \mathrm{ppm}$ respectively. The method is robust as in robustness parameter deliberate changes were made for which individual and cumulative RSD values for each set were less than $5.0 \%$.

\section{Conclusions:-}

Headspace gas chromatographic method developed can be successfully applied for quantitative determination of Acetone, Acetonitrile and Ethyl acetate in Neostigmine Methylsulfate bulk drug sample on a manufacturing level in the pharmaceutical industry. The method is validated and found out to be specific, linear, accurate, precise and robust, Acceptable data for all method validation parameters tested and found out to be satisfactory.

\section{Acknowledgment:-}

The authors wish to thank the management of Indoco Remedies Ltd. for supporting this work. The authors wish to acknowledge the process research department for providing the samples for this research.

\section{Refrences:-}

1. M. B. Walker, "Case showing the effect of prostigmin on myasthenia gravis," Proceedings of the Royal Society of Medicine, vol. 28, no. 6, pp. 759-761, 1935.

2. R. N. Banerjee, A. L. Sahni, K. A. Chacko, and K. Vijay, "Neostigmine in the treatment of Elapidae bites," The Journal of the Association of Physicians of India, vol. 20, no. 7, pp. 503-509, 1972.

3. G. S. Murphy, J. W. Szokol, J. S. Vender, J. H. Marymont, and M. J. Avram, "The use of neuromuscular blocking drugs in adult cardiac surgery: results of a national postal survey," Anesthesia and Analgesia, vol. 95, no. 6, pp. 1534-1539, 2002.

4. M. M. Mehndiratta, S. Pandey, and T. Kuntzer, "Acetylcholinesterase inhibitor treatment for myasthenia gravis," Cochrane Database of Systematic Reviews, vol. 2, Article ID CD006986, 2011.

5. Qin $\mathrm{L}, \mathrm{Hu} \mathrm{CQ}$, Yin LH. Establishment of a knowledge base for prescreening residual solvents in pharmaceuticals. Chromatographia. 2004 Apr 1;59(7-8):475-80.

6. B'hymer C. Residual solvent testing: a review of gas-chromatographic and alternative techniques. Pharmaceutical research. 2003 Mar 1;20(3):337-44.

7. Otero R, Carrera G, Dulsat JF, Fábregas JL, Claramunt J. Static headspace gas chromatographic method for quantitative determination of residual solvents in pharmaceutical drug substances according to European Pharmacopoeia requirements. Journal of Chromatography A. 2004 Nov 19;1057(1):193-201.

8. Dwivedi AM. Residual solvent analysis in pharmaceuticals. Pharmaceutical technology. 2002 Nov;26(11):42-7. 
9. Cheng C, Liu S, Mueller BJ, Yan Z. A generic static headspace gas chromatography method for determination of residual solvents in drug substance. Journal of Chromatography A. 2010 Oct 8;1217(41):6413-21.

10. Guideline, ICH. Validation of analytical procedures: text and methodology. Q2 (R1) 1 (2005) Nov.

11. Guideline ICH. Impurities: Guideline for residual solvents Q3C (R5). Current Step. 2005 Nov;4:509.

12. Swartz ME, Krull IS, editors. Analytical method development and validation. CRC Press; 1997 May 16.

13. Kazakevich YV, Lobrutto R. HPLC for pharmaceutical scientists. John Wiley \& Sons; 2007 Feb 16.

14. Snyder, Lloyd R., Joseph J. Kirkland, and Joseph L. Glajch. Practical HPLC method development. John Wiley \& Sons, 2012.

15. Chan, Chung Chow, et al., eds. Analytical method validation and instrument performance verification. John Wiley \& Sons, 2004.

16. Ermer, Joachim, and John H. McB Miller, eds. Method validation in pharmaceutical analysis: A guide to best practice. John Wiley \& Sons, 2006. 